\title{
Image Transmission Technique via Mosaic Image Steganography
}

\author{
Athira Leeladharan ${ }^{1}$, A. S. Vibith ${ }^{2}$ \\ ${ }^{1}$ ME-CSE, Kingston Engineering College, Vellore, India \\ ${ }^{2}$ Assistant Professor -CS, Kingston Engineering College, Vellore, India
}

\begin{abstract}
A new secure image transmission technique is proposed, which transforms automatically a given large-volume secret image into a so-called secret-fragment-visible mosaic image of the same size. The mosaic image, which looks similar to an arbitrarily selected target image and may be used as a camouflage of the secret image, is yielded by dividing the secret image into fragments and transforming their color characteristics to be those of the corresponding blocks of the target image. Skillful techniques are designed to conduct the color transformation process so that the secret image may be recovered nearly lossless.
\end{abstract}

Keywords: Mosaic Image, Steganography, Color transformation, Secure Image Transmission

\section{Introduction}

With the rapid development of Internet Technology, people can communicate with each other easily. They can transmit and share any type of data conveniently through Internet. During data transmission through a network, the security of data is a major concern. With the rapid development of multimedia and network/technologies, the security of multimedia becomes more and more important, since multimedia data are transmitted over open networks more and more frequently. Typically, reliable security is necessary to content protection of digital images and videos.

\section{Problem Characterization}

With the rapid development of Internet, people find it very easy to transmit digital information. But the communication through an open network creates many security issues. Many techniques have been implemented in the recent world in order to face this challenge.

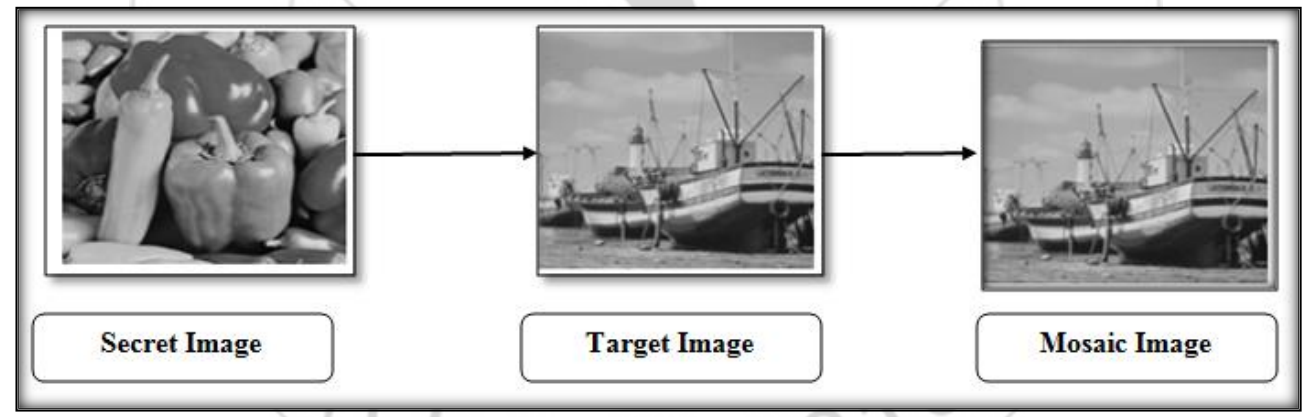

In most of the techniques, a common problem found is the randomness in form of the resultant images. Since encryption is performed in data (text or images), the resultant data is a meaningless file. This will attract an attacker who can try all possible ways to get the secret information. This was found to be a major threat to security.

\section{Objective of the Paper}

The goal of this paper is to overcome the above mentioned security issue by the idea of a new technique called Secure Image Transmission via Mosaic Image Steganography. The main objective of this technique is to solve the first security issue mentioned above, that is, randomness in the resultant image. The secret image is converted into a mosaic image by applying certain transformations. The mosaic image looks similar to a pre-selected target image. Hence an attacker does not have any chance of mistaking the image as a secret data carrier.

\section{Features of Mosaic Image Steganograph}

The Mosaic Image Steganography has a rich set of features. It includes;

1) Mosaic is a type of artwork created by composing small pieces of materials, such as stone, glass, tile, etc.

2) Invented in ancient time, they are still used in many applications today.

3) Creation of Mosaic images by computer are a new research direction in recent years.

4) Taxonomy of mosaic images into four types is proposed, including crystallization mosaic, ancient mosaic, photomosaic and puzzle image mosaic.

5) The first two types are obtained from decomposing a source image into tiles (with different colors, sizes, and rotations) and reconstructing the image by properly painting the tiles, and so they both may be called tile mosaics.

6) The other two types of mosaics are obtained by fitting images from a database to cover an assigned source image, and both may be called multi-picture mosaics. 


\section{International Journal of Science and Research (IJSR)}

ISSN (Online): 2319-7064

Index Copernicus Value (2013): 6.14 | Impact Factor (2015): 6.391

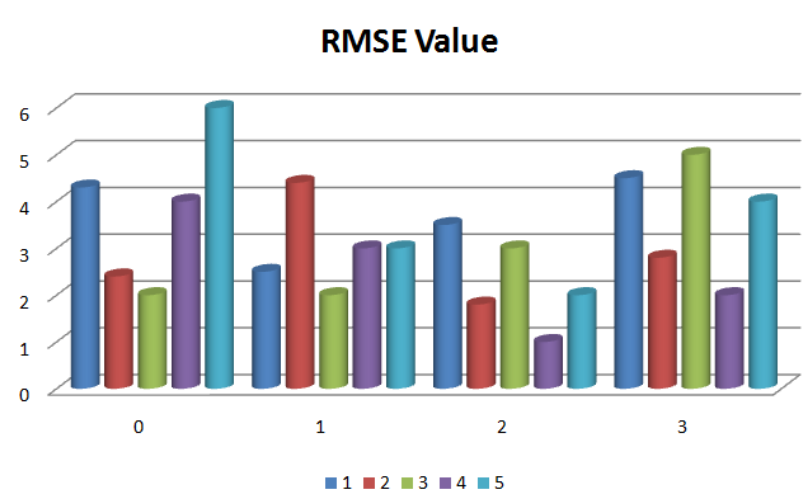

5. Literature Survey

A body of literature has been conducted by several authors and a list of them is given below;

\section{By I-Jen Lai and Wen-Hsiang Tsai}

In this paper, a new type of computer art image called secret-fragment- visible mosaic image is proposed, which is created automatically by composing small fragments of a given image to become a target image in a mosaic form, achieving an effect of embedding the given image visibly but secretly in the resulting mosaic image. This effect of information hiding is useful for covert communication or secure keeping of secret images. To create a mosaic image of this type from a given secret color image, the 3-D color space is transformed into a new 1-D color scale, based on which a new image similarity measure is proposed for selecting from a database a target image that is the most similar to the given secret image. A fast greedy search algorithm is proposed to find a similar tile image in the secret image to fit into each block in the target image. The information of the tile image fitting sequence is embedded into randomly-selected pixels in the created mosaic image by a lossless LSB replacement scheme using a secret key; without the key, the secret image cannot be recovered.

\section{By X. Li, B. Yang, and T. Zeng}

Prediction-error expansion (PEE) is an important technique of reversible watermarking which can embed large payloads into digital images with low distortion. In this paper, the PEE technique is further investigated and an efficient reversible watermarking scheme is proposed, by incorporating in PEE two new strategies, namely, adaptive embedding and pixel selection. Unlike conventional PEE which embeds data uniformly, we propose to adaptively embed 1 or 2 bits into expandable pixel according to the local complexity. This avoids expanding pixels with large prediction-errors, and thus, it reduces embedding impact by decreasing the maximum modification to pixel values. Meanwhile, adaptive PEE allows very large payload in a single embedding pass, and it improves the capacity limit of conventional PEE. Also select pixels of smooth area for data embedding and leave rough pixels unchanged. In this way, compared with conventional PEE, a more sharply distributed prediction-error histogram is obtained and a better visual quality of watermarked image is observed.

3. By X. Hu, W. Zhang, X. Hu, N. Yu, X. Zhao, and F. Li Recently, code construction approaching the rate-distortion bound of reversible data hiding has been proposed by Lin , in which the coding/decoding process needs the optimal probability distribution of marked-signals as parameters. Therefore, the efficiency and accuracy of estimating the optimal marked-signal distribution will greatly influence the speeds of encoding and decoding. In this paper, a fast algorithm is proposed to solve the optimal marked-signal distribution. Furthermore, modify the method to achieve the optimal distribution directly according to a given distortion constraint or an expected embedding rate, which makes it more practical for applications.

\section{By W.-H. Lin, S.-J. Horng, T.-W. Kao, P. Fan, C.-L.} Lee and Y. Pan

This paper proposes a blind watermarking algorithm based on the significant difference of wavelet coefficient quantization for copyright protection. Every seven non overlap wavelet coefficients of the host image are grouped into a block. The largest two coefficients in a block are called significant coefficients in this paper and their difference is called significant difference. Quantize the local maximum wavelet coefficient in a block by comparing the significant difference value in a block with the average significant difference value in all blocks. The maximum wavelet coefficients are so quantized that their significant difference between watermark bit 0 and watermark bit 1 exhibits a large energy difference which can be used for watermark extraction. During the extraction, an adaptive threshold value is designed to extract the watermark from the watermarked image under different attacks. We compare the adaptive threshold value to the significant difference which was quantized in a block to determine the watermark bit. The experimental results show that this method is quite effective against JPEG compression, low-pass filtering, and Gaussian noise; the PSNR value of a watermarked image is greater than $40 \mathrm{~dB}$.

\section{By S. Lee, C. D. Yoo, and T. Kalker}

This paper proposes a high capacity reversible image watermarking scheme based on integer to integer wavelet transforms. The scheme divides an input image into nonoverlapping blocks and embeds a watermark into the highfrequency wavelet coefficients of each block. The conditions to avoid both underflow and overflow in the spatial domain are embedded includes not only messages but also side information used to reconstruct the exact original image. To minimize the mean squared distortion between the original and the watermarked images given a payload, the watermark is adaptively embedded into the image. The experimental results show that the technique achieves higher embedding capacity while maintaining distortion at a lower level than the existing reversible watermarking schemes.

\section{By Z. Ni, Y. Q. Shi, N. Ansari and W. Su}

A novel reversible data hiding algorithm, which can recover the original image without any distortion from the marked image after the hidden data have been extracted, is presented in this paper. This algorithm utilizes the zero or the minimum points of the histogram of an image and slightly modifies the pixel grayscale values to embed data into the image. It can embed more data than many of the existing reversible data hiding algorithms. It is proved analytically and shown experimentally that the peak signal-to-noise ratio (PSNR) of the marked image generated by this method 


\section{International Journal of Science and Research (IJSR) \\ ISSN (Online): 2319-7064}

Index Copernicus Value (2013): 6.14 | Impact Factor (2015): 6.391

versus the original image is guaranteed to be above $48 \mathrm{~dB}$ This lower bound of PSNR is much higher than that of all reversible data hiding techniques reported in the literature. The computational complexity of our proposed technique is low and the execution time is short. The algorithm has been successfully applied to a wide range of images, including commonly used images, medical images, texture images, aerial images and all of the 1096 images in CorelDraw database.

\section{By J. Tian}

Reversible data embedding has drawn lots of interest recently. Being reversible, the original digital content can be completely restored. The paper present a novel reversible data-embedding method for digital images. It explores the redundancy in digital images to achieve very high embedding capacity, and keep the distortion low.

\section{By Y. Hu, H.-K. Lee, K. Chen, and J. Li}

Current difference-expansion (DE) embedding techniques perform one layer embedding in a difference image. They do not turn to the next difference image for another layer embedding unless the current difference image has no expandable differences left. The obvious disadvantage of these techniques is that image quality may have been severely degraded even before the later layer embedding begins because the previous layer embedding has used up all expandable differences, including those with large magnitude. Based on integer Haar wavelet transform, we propose a new DE embedding algorithm, which utilizes the horizontal as well as vertical difference images for data hiding. The paper introduces a dynamical expandable difference search and selection mechanism. This mechanism gives even chances to small differences in two difference images and effectively avoids the situation that the largest differences in the first difference image are used up while there is almost no chance to embed in small differences of the second difference image. Also present an improved histogram-based difference selection and shifting scheme, is presented which refines our algorithm and makes it resilient to different types of images.

9. By W. Zhang, X. Hu, X. Li, and N. Yu

State-of-the-art schemes for reversible data hiding (RDH) usually consist of two steps: first construct a host sequence with a sharp histogram via prediction errors, and then embed messages by modifying the histogram with methods, such as difference expansion and histogram shift. In this paper, we focus on the second stage, and propose a histogram modification method for RDH, which embeds the message by recursively utilizing the decompression and compression processes of an entropy coder. We prove that, for independent identically distributed (i.i.d.) gray-scale host signals, the proposed method asymptotically approaches the rate-distortion bound of $\mathrm{RDH}$ as long as perfect compression 9; can be realized, i.e., the entropy coder can approach entropy. Therefore, this method establishes the equivalency between reversible data hiding and lossless data compression. Experiments show that this coding method can be used to improve the performance of previous $\mathrm{RDH}$ schemes and the improvements are more significant for larger images
10. By V. Sachnev, H. J. Kim, J. Nam, S. Suresh, and Y.-Q Shi.

This paper presents a reversible or lossless watermarking algorithm for images without using a location map in most cases. This algorithm employs prediction errors to embed data into an image. A sorting technique is used to record the prediction errors based on magnitude of its local variance. Using sorted prediction errors and, if needed, though rarely, a reduced size location map allows us to embed more data into the image with less distortion.

\section{Conclusion and Future Enhancement}

Specifically, after a target image is selected arbitrarily, the given secret image is first divided into rectangular fragments called tile images, which then are fit into similar blocks in the target image, called target blocks, according to a similarity criterion based on color variations. Next, the color characteristic of each tile image is transformed to be that of the corresponding target block in the target image, resulting in a mosaic image which looks like the target image. Relevant schemes are also proposed to conduct nearly lossless recovery of the original secret image from the resulting mosaic image. The proposed method is new in that a meaningful mosaic image is created, in contrast with the image encryption method that only creates meaningless noise images. Also, the proposed method can transform a secret image into a disguising mosaic image without compression, while a data hiding method must hide a highly compressed version of the secret image into a target image when the secret image and the target image have the same data volume.

\section{Acknowledgement}

I would like to take this opportunity to express my profound gratitude and deep regard to my guide, Prof.A.S.Vibith CSE, Kingston Engineering College, for his exemplary guidance, valuable feedback and constant encouragement in completing this paper. His valuable suggestions were of immense help in getting this work done. Working under him, was an extremely knowledgeable experience. Also, I would like to extend my sincere gratitude to my husband and my parents for their constant support and encouragement in completing this paper.

\section{References}

[1] J. Fridrich, "Symmetric ciphers based on twodimensional chaotic maps," Int. J. Bifurcat. Chaos, vol. 8, no. 6, pp. 1259-1284, 1998.

[2] G. Chen,Y. Mao, and C. K. Chui, "A symmetric image encryption scheme based on 3D chaotic cat maps," Chaos Solit. Fract., vol. 21, no. 3, pp. 749-761, 2004.

[3] L. H. Zhang, X. F. Liao, and X. B. Wang, "An image encryption approach based on chaotic maps," Chaos Solit. Fract., vol. 24, no. 3, pp. 759-765, 2005.

[4] H. S. Kwok and W. K. S. Tang, "A fast image encryption system based on chaotic maps with finite precision representation," Chaos Solit. Fract., vol. 32, no. 4, pp. 1518-1529, 2007. 


\section{International Journal of Science and Research (IJSR) \\ ISSN (Online): 2319-7064}

Index Copernicus Value (2013): 6.14 | Impact Factor (2015): 6.391

[5] S. Behnia, A. Akhshani, H. Mahmodi, and A. Akhavan, "A novel algorithm for image encryption based on mixture of chaotic maps," Chaos Solit. Fract., vol. 35, no. 2, pp. 408-419, 2008.

[6] D. Xiao, X. Liao, and P. Wei, "Analysis and improvement of a chaosbased image encryption algorithm," Chaos Solit. Fract., vol. 40, no. 5, pp. 21912199, 2009.

[7] V. Patidar, N. K. Pareek, G. Purohit, and K. K. Sud, "A robust and secure chaotic standard map based pseudorandom permutationsubstitution scheme for image encryption," Opt. Commun., vol. 284, no. 19, pp. 4331-4339, 2011.

[8] C. K. Chan and L. M. Cheng, "Hiding data in images by simple LSB substitution," Pattern Recognit.., vol. 37, pp. 469-474, Mar. 2004.

[9] Z. Ni, Y. Q. Shi, N. Ansari, and W. Su, "Reversible data hiding," IEEE Trans. Circuits Syst. Video Technol., vol. 16, no. 3, pp. 354-362, Mar. 2006.

[10] J. Tian, "Reversible data embedding using a difference expansion," IEEE Trans. Circuits Syst. Video Technol., vol. 13, no. 8, pp. 890-896, Aug. 2003.

[11] Y. Hu, H.-K. Lee, K. Chen, and J. Li, "Difference expansion based reversible data hiding using two embedding directions," IEEE Trans. Multimedia, vol. 10, no. 8, pp. 1500-1512, Dec. 2008.

[12] V. Sachnev, H. J. Kim, J. Nam, S. Suresh, and Y.-Q. Shi, "Reversible watermarking algorithm using sorting and prediction," IEEE Trans. Circuits Syst. Video Technol., vol. 19, no. 7, pp. 989-999, Jul. 2009.

[13]X. Li, B. Yang, and T. Zeng, "Efficient reversible watermarking based on adaptive prediction-error expansion and pixel selection," IEEE Trans. Image Process., vol. 20, no. 12, pp. 3524-3533, Dec. 2011.

[14] W. Zhang, X. Hu, X. Li, and N. Yu, "Recursive histogram modi- fication: Establishing equivalency between reversible data hiding and lossless data compression," IEEE Trans. Image Process., vol. 22, no. 7, pp. 2775-2785, Jul. 2013.

[15]J. Fridrich, M. Goljan, and R. Du, "Invertible authentication,” Proc. SPIE, vol. 3971, 2001, pp. 197208.

[16]C. C. Chang, C. C. Lin, C. S. Tseng, and W. L. Tai, "Reversible hiding in DCT-based compressed images," Inf. Sci., vol. 177, no. 13, pp. 2768-2786, 2007.

[17] S. Lee, C. D. Yoo, and T. Kalker, "Reversible image watermarking based on integer-to-integer wavelet transform," IEEE Trans. Inf. Forens. Secur., vol. 2, no. 3, pp. 321-330, Sep. 2007.

[18] W.-H. Lin, S.-J. Horng, T.-W. Kao, P. Fan, C.-L. Lee, and Y. Pan, "An efficient watermarking method based on significant difference of wavelet coefficient quantization," IEEE Trans. Multimedia, vol. 10, no. 5, pp. 746-757, Aug. 2008. 